\title{
Influence du contexte expérimental sur l'interprétation des anaphores pronominales en français
}

\author{
Saveria Colonna ${ }^{1}$, Sarah Schimke ${ }^{2}$, Coralie Vincent ${ }^{3}$, Israel de la Fuente ${ }^{4}$ et Barbara Hemforth ${ }^{4}$ \\ ${ }^{1}$ Laboratoire Structures Formelles du Langage (CNRS \& Université Paris 8) \\ ${ }^{2}$ Université de Münster \\ ${ }^{3}$ Laboratoire Structures Formelles du Langage (CNRS \& Université Paris 8) \\ ${ }^{4}$ Laboratoire de Linguistique Formelle (CNRS \& Université Paris Diderot)
}

\begin{abstract}
Résumé. Cette étude présente les résultats de trois questionnaires réalisés en français afin d'observer les préférences d'interprétation de formes pronominales ambiguës. Plus précisément, nous nous sommes intéressés à l'influence du contexte expérimental sur l'interprétation de pronoms anaphoriques plus ou moins réduits. Dans un premier questionnaire, seules des constructions avec le pronom faible « il » étaient présentées. Un deuxième questionnaire comportait seulement la forme forte « lui, il ». Enfin, dans un troisième questionnaire les deux formes étaient mélangées afin d'observer si la présence, dans un même questionnaire, des deux formes (« il » et « lui, il ») pouvait influencer leur interprétation. C'est seulement lorsque les deux formes sont présentées dans un même questionnaire que nous observons une division fonctionnelle entre la forme pronominale réduite « il » et la forme accentuée « lui, il ». La présence de « lui, il » dans le même questionnaire que le pronom « il », augmente les interprétations du pronom " il » en faveur du référent saillant (premier mentionné, sujet et topique) et les interprétations de « lui, il » en faveur de l'antécédent moins saillant. Ces résultats révèlent à quel point les locuteurs sont rapidement capables d'adapter leur préférence d'interprétation à la présence de formes alternatives dans le contexte.
\end{abstract}

\begin{abstract}
This paper presents the results of three questionnaires in which we investigated interpretational preferences for ambiguous pronominal forms in French. In particular, we examined the influence of the experimental context on the interpretation of more or less reduced anaphoric pronominal forms. In the first questionnaire, only constructions with the clitic pronoun "il" were tested. In the second questionnaire, only constructions with the strong form "lui, il" were tested. Finally, in the third questionnaire, both forms were mixed in order to test whether the presence of the two forms ("il" and "lui, il") in a single questionnaire influences their interpretation. A division of labour in the processing of the reduced form "il" and of the strong one "lui, il" was observed only in the third questionnaire, i.e. when both forms were presented together. The presence of the form "lui, il" in the same questionnaire as the pronoun "ill" increased the interpretations of the pronoun "il" in favour of the more salient referent (i.e., first mentioned, subject and topic) and the interpretations of "lui, il" in favour of the less salient antecedent. These results reveal that comprehenders can and do adapt their interpretational preferences to the presence of alternative forms in the context.
\end{abstract}




\section{Introduction}

Pendant la compréhension du langage, les expressions référentielles telles que les anaphores pronominales sont rapidement et correctement reliées $\mathrm{au}(\mathrm{x})$ référent(s) du discours au(x)quel(s) elles renvoient et ce, sans difficulté (du moins dans la plupart des cas). Ceci soulève la question de savoir comment les anaphores sont reliées à leurs antécédents. La plupart des modèles de la résolution d'anaphores s'accordent sur le fait que les formes les plus réduites sont préférentiellement interprétées comme se référant à un antécédent saillant dans le discours au contraire des formes moins réduites (Ariel, 1990 ; Givón, 1983 ; Gundel, Hedberg \& Zacharski, 1993). De même, la plupart des modèles s'accordent sur le fait que plusieurs facteurs déterminent la saillance d'un référent. Parmi les facteurs fréquemment mentionnés, on peut citer : la récence linéaire, la structure syntaxique des énoncés, le sémantisme de certains verbes et le statut informationnel des référents potentiels (pour une revue récente, voir Frazier, 2012 ; de la Fuente, Hemforth, Colonna \& Schimke, sous presse). En revanche, la pondération de ces différents facteurs suivant le contexte et la langue est toujours débattue.

Dans ce papier, nous nous intéressons à l'influence du contexte expérimental sur l'interprétation de pronoms anaphoriques plus ou moins réduits en français. Avant d'exposer nos hypothèses sur le français, nous commencerons par présenter les travaux sur l'interprétation des pronoms dans les langues pro-drop qui ont inspiré notre manipulation.

\subsection{Travaux antérieurs sur les langues pro-drop}

Dans les langues pro-drop, différents linguistes ont proposé une division du travail entre pronoms zéro et pronoms ouverts : la forme nulle serait utilisée pour se référer à l'antécédent le plus saillant dans le contexte alors que la forme ouverte serait utilisée pour se référer à un antécédent moins saillant (Luján, 1985, 1986 ; Carminati, 2002, 2005). Leurs propositions diffèrent cependant quant à ce qui détermine la saillance des antécédents. Luján $(1985,1986)$ propose que dans les langues pro-drop, la forme nulle du pronom est utilisée pour se référer au topique du discours, alors que la forme ouverte du pronom est utilisée pour signaler un changement de topique et se réfère donc à une entité moins saillante. Carminati (2002, 2005) propose l'hypothèse de la position de l'antécédent (PAH) suivant laquelle les pronoms nuls, au contraire des pronoms ouverts, se réfèrent préférentiellement à l'antécédent syntaxiquement saillant

(i.e., le sujet syntaxique). Pour Luján, c'est donc le statut discursif des antécédents qui détermine leur saillance alors que pour Carminati, c'est leur position syntaxique. Les deux propositions conduisent (du moins dans les cas où sujet et topique sont confondus) aux mêmes prédictions : un pronom zéro (au contraire d'un pronom ouvert) sera préférentiellement interprété comme se référant à l'antécédent sujet et topique. Cette prédiction a été confirmée en italien (Carminati, 2002 ; Filiaci, 2010 ; Filiaci, Sorace \& Carreiras, 2013) et, du moins en partie, en espagnol (Alonso-Ovalle, Fernandes-Solera, Frazier \& Clifton, 2002 ; Filiaci, 2010 ; Filiaci et al., 2013 ; Jegerski, VanPatten \& Keating, 2011 ; Keating, VanPatten \& Jegerski, 2011) et en portugais européen (Costa, Faria \& Kail, 2004 ; Costa, 2010). Dans une expérience de lecture en auto-présentation segmentée de phrases telles que (1), Carminati (2002) a observé que, conformément aux prédictions de la $\mathrm{PAH}$, la seconde proposition était lue plus lentement lorsque le pronom nul renvoyait à l'antécédent objet (1d) que lorsqu'il renvoyait à l'antécédent sujet (1b), et lorsque le pronom ouvert renvoyait à l'antécédent sujet (1a) que lorsqu'il renvoyait à l'antécédent objet (1c). 
(1) Quando Maria è andata a trovare Vanessa in ospedale,

a. lei le ha portato un mazzo di fiori.

b. Ø le ha portato un mazzo di fiori.

c. lei era già fuori pericolo.

d. Ø era già fuori pericolo.

'Quand Marie est allée voir Vanessa à l'hôpital, (elle) lui a apporté un bouquet de fleurs/(elle) était déjà hors de danger.'

En espagnol, Alonso-Ovalle et al. (2002) ont testé les hypothèses de Carminati dans un questionnaire. Ils ont observé les préférences d'interprétation d'un pronom sujet ambigu dans des phrases telles que (2). Les résultats montrent une préférence pour le premier référent mentionné (et sujet) pour le pronom zéro (2b) et pas de préférence pour le pronom ouvert (2a). Comme l'étude d'Alonso-Ovalle et collaborateurs, les autres études menées en espagnol ont révélé une préférence pour l'antécédent syntaxiquement saillant (le sujet) avec un pronom zéro mais pas de préférence claire avec un pronom ouvert, ne répliquant qu'en partie les résultats de Carminati en italien (Filiaci, 2010 ; Filiaci et al., 2013 ; Jegerski et al., 2011 ; Keating et al., 2011).
a. Juan pegó a Pedro. Él está enfadado.
b. Juan pegó a Pedro. Ø Está enfadado.
'Jean a frappé Pierre. (Il) est en colère.'

En portugais européen, Costa, Faria et Kail (2004) ont réalisé une tâche de détection de sonde dans des phrases telles que (3). Les participants devaient décider le plus rapidement possible si une sonde (soit le sujet, soit l'objet de la proposition principale) présentée après le verbe de la proposition subordonnée (comme illustré en [3a]) correspondait ou pas à un mot de la phrase qu'ils sont en train de lire. Les auteurs observent que les temps de réaction varient en fonction de la forme du pronom (nulle vs ouverte) et du sémantisme du verbe dans la proposition principale. Le verbe dans la proposition principale était soit un verbe à causalité implicite, soit un verbe sans biais causal. Un verbe dit «à causalité implicite » est un verbe transitif avec deux arguments animés qui présente la caractéristique de déclencher systématiquement une explication en faveur d'un des deux arguments (voir e.g., Brown \& Fish, 1983). Par exemple, dans (3), le verbe admirar (admirer) est biaisé en faveur de l'objet.
a. No congresso,/ a Maria/ admirou/ a Sofia/ porque/ ela/ fez/ Maria/ um bom discurso.
b. No congresso, a Maria admirou a Sofia porque $\emptyset$ fez um bom discurso.

'Au congrès, Maria admirait Sofia parce qu'(elle) a fait une bonne présentation.'

Avec des verbes sans biais de causalité implicite, le temps de détection de la sonde lorsqu'elle correspond au sujet de la proposition principale (ici, Maria) est plus long après un pronom ouvert qu' après un pronom 
nul, suggérant que le pronom ouvert est préférentiellement interprété comme se référant à l'antécédent moins saillant (i.e., l'objet de la principale). En revanche, avec des verbes biaisés en faveur de l'objet ou du sujet, le temps pour détecter la sonde (Maria) ne diffère pas suivant que le pronom est réalisé (3a) ou pas (3b). Costa et al. (2004) montrent ainsi que la division du travail observée dans les langues pro-drop peut être fortement influencée par des facteurs sémantiques tels que la causalité implicite.

Pour résumer, même si les études sur l'interprétation des pronoms dans les langues pro-drop révèlent des différences suivant la langue étudiée et la méthode utilisée, toutes s'accordent sur le fait que formes nulles et formes ouvertes ne montrent pas les mêmes préférences, et concluent à un partage du travail entre les deux formes. Il faut cependant noter que dans toutes les expériences sus-citées, pronoms nuls et pronoms ouverts, pour être comparés, étaient présentés ensemble. Or, de la Fuente et Hemforth (2013) ont montré qu'en espagnol, le fait de présenter dans une même expérience pronoms nuls et pronoms ouverts pouvait accentuer la division du travail prédite entre ces deux formes. En effet, dans un questionnaire où ils ne présentaient que des pronoms nuls dans des phrases telles que (4b), ils n'ont pas observé la préférence attendue pour l'antécédent syntaxiquement saillant (le sujet). Alors que dans un questionnaire où pronoms nuls et pronoms ouverts étaient mélangés, ils ont observé que les pronoms nuls (4b) étaient préférentiellement interprétés comme se référant à l'antécédent en position sujet et les pronoms ouverts (4a) comme se référant à l'antécédent en position objet. De la Fuente et Hemforth (2013) concluent que la division du travail entre pronoms nuls et pronoms ouverts observée dans des expériences présentant les deux formes est au moins en partie due à des stratégies métalinguistiques développées consciemment par les participants durant l'expérience.

(4) a. Eduardo llamó a Samuel cuando él estaba en la oficina.

b. Eduardo llamó a Samuel cuando Ø estaba en la oficina.

'Edouard a appelé Samuel quand (il) était dans le bureau.'

\subsection{Hypothèses sur le français}

Nous avons voulu savoir dans quelle mesure les stratégies observées dans les langues pro-drop étaient appliquées dans une langue avec un pronom sujet faible telle que le français. En particulier, nous avons voulu observer si l'interprétation du pronom anaphorique «il » pouvait être influencée par la présence dans le contexte expérimental d'un autre marqueur anaphorique ; de la même manière que l'interprétation du pronom nul en espagnol est influencée par la présence de pronoms pleins dans le contexte expérimental (de la Fuente \& Hemforth, 2013).

En français (surtout en français oral), on peut accentuer le pronom sujet faible «il » en utilisant le pronom accentué «lui » suivi du pronom «il» comme en (5b). Si les deux constructions (5a) et (5b) sont possibles, elles ne sont pas équivalentes. Nous avons vu en effet que tous les marqueurs anaphoriques ne véhiculaient pas la même instruction de traitement. En français, Fossard et Rigalleau (2005) ont par exemple montré que le pronom anaphorique «il/elle » se référait à l'entité la plus saillante du discours alors que le pronom démonstratif « celui-ci/celle-là » était préférentiellement interprété comme se référant à un antécédent moins saillant. De même, nous pouvons facilement faire l'hypothèse que les formes «il » et «lui, il » ne véhiculent pas la même instruction de traitement. Le pronom «il » en (5a) devrait être préférentiellement relié à l'antécédent le plus saillant (ici, Pierre). En effet, en plus d'être le premier référent mentionné, il est aussi sujet, agent et topique. Autant de raisons pour prédire qu'il est saillant et qu'il sera l'antécédent préféré du pronom anaphorique faible «il » dans (5a). En revanche, l'emploi de la forme contrastive «lui, il » pouvant être utilisée pour signaler un changement de topique (voir e.g., van Kampen, 2008), «lui, il» en (5b) devrait être préférentiellement relié au référent moins saillant (ici, Jean).
a. Pierre a giflé Jean. Il était stagiaire.
b. Pierre a giflé Jean. Lui, il était stagiaire. 
La question que nous posons ici est de savoir si les instructions de traitement associées aux différentes formes anaphoriques diffèrent suivant si une ou plusieurs formes sont présentes dans le contexte expérimental. Nous faisons l'hypothèse que la co-occurrence d'une forme réduite avec une forme moins réduite (respectivement, «il » et « lui, il ») accentuera leur division fonctionnelle : la forme réduite sera d'autant plus interprétée comme se référant à l'entité saillante du discours, tandis que la forme moins réduite sera d'autant plus interprétée comme se référant à une entité moins saillante.

Pour répondre à cette question, nous avons réalisé trois questionnaires en français. Dans un premier questionnaire, seules des constructions avec «il » étaient présentées, ce qui nous permettait de connaître la préférence de base pour le pronom «il » dans des énoncés tels que (5a). Un deuxième questionnaire comportait la seule forme « lui, il » avec des énoncés tels que $(5 b)$. Enfin, dans un troisième questionnaire les deux formes étaient mélangées afin d’observer si la présence, dans un même questionnaire, des deux formes («il » et « lui, il ») pouvait influencer leur interprétation.

\section{Questionnaires}

\subsection{Méthode}

\subsubsection{Participants}

Cinquante-huit participants ont volontairement complété le Questionnaire 1, 39 participants le Questionnaire 2 et 50 participants le Questionnaire 3. Tous les participants étaient de langue maternelle française et chaque participant n'a participé qu'à un seul questionnaire.

\subsubsection{Matériel}

Nous avons construit 28 items expérimentaux sur le modèle des énoncés présentés en (5). Chaque item était composé de deux phrases de la même forme : le verbe dans la première phrase était un verbe d'action avec un sujet agent et un objet patient. La causalité implicite associée aux verbes n'a pas été systématiquement contrôlée. Tous les sujets et les objets étaient des noms propres masculins ii . La seconde phrase contenait un sujet pronominalisé ambigu qui pouvait se référer soit au sujet, soit à l'objet de la première phrase. Chaque couple de phrases était suivi d'une phrase dont le début était à compléter par les participants (comme dans les exemples $5 \mathrm{a}$ et $5 \mathrm{~b}$ reproduits ci-dessous). Les préférences d'interprétation du pronom ambigu étaient ainsi observées, le complètement de la phrase nécessitant l'interprétation du pronom.

Dans le Questionnaire 1, la seconde phrase commençait toujours par le pronom sujet «il», comme en (5a). Dans le Questionnaire 2, la seconde phrase commençait par la forme pronominale accentuée «lui, il » comme en (5b). Dans le Questionnaire 3, les deux conditions « il » et « lui, il » étaient mélangées. Deux versions du Questionnaire 3 ont été construites afin que les participants ne voient pas les mêmes items dans les deux conditions. Il y avait quatorze items par condition dans chaque version du Questionnaire 3.

a. Pierre a giflé Jean. Il était stagiaire.

était stagiaire.

b. Pierre a giflé Jean. Lui, il était stagiaire.

était stagiaire.

Dans les trois questionnaires, les items expérimentaux étaient mélangés à vingt-quatre items de remplissage. Ces items de remplissage contenaient tous une proposition principale et une proposition relative ambiguë, la proposition principale introduisait deux antécédents potentiels de la relative dans un 
syntagme nominal complexe. L'énoncé à compléter portait sur l'antécédent de la relative comme dans l'exemple (6).

(6) Léa est fiancée au voisin du cordonnier qui danse.

danse.

\subsubsection{Procédure}

Les questionnaires ont été implémentés sur la plateforme IbexFarm (Drummond, 2010) pour être proposés en ligne. Les participants avaient pour consigne de lire attentivement les phrases et de compléter les énoncés incomplets le plus spontanément possible. Les items apparaissaient à l'écran un à un sans qu'il soit possible de revenir en arrière. Le complètement d'un questionnaire prenait une quinzaine de minutes. Les réponses étaient codées suivant que les participants choisissaient le premier (N1) ou le deuxième nom (N2) mentionné comme antécédent du pronom.

\subsection{Résultats}

La Figure 1 représente les pourcentages de choix pour N2 dans les trois questionnaires, suivant le type de forme pronominale («il »vs « lui, il ») et le type de questionnaire (avec une seule forme $v s$ deux formes).

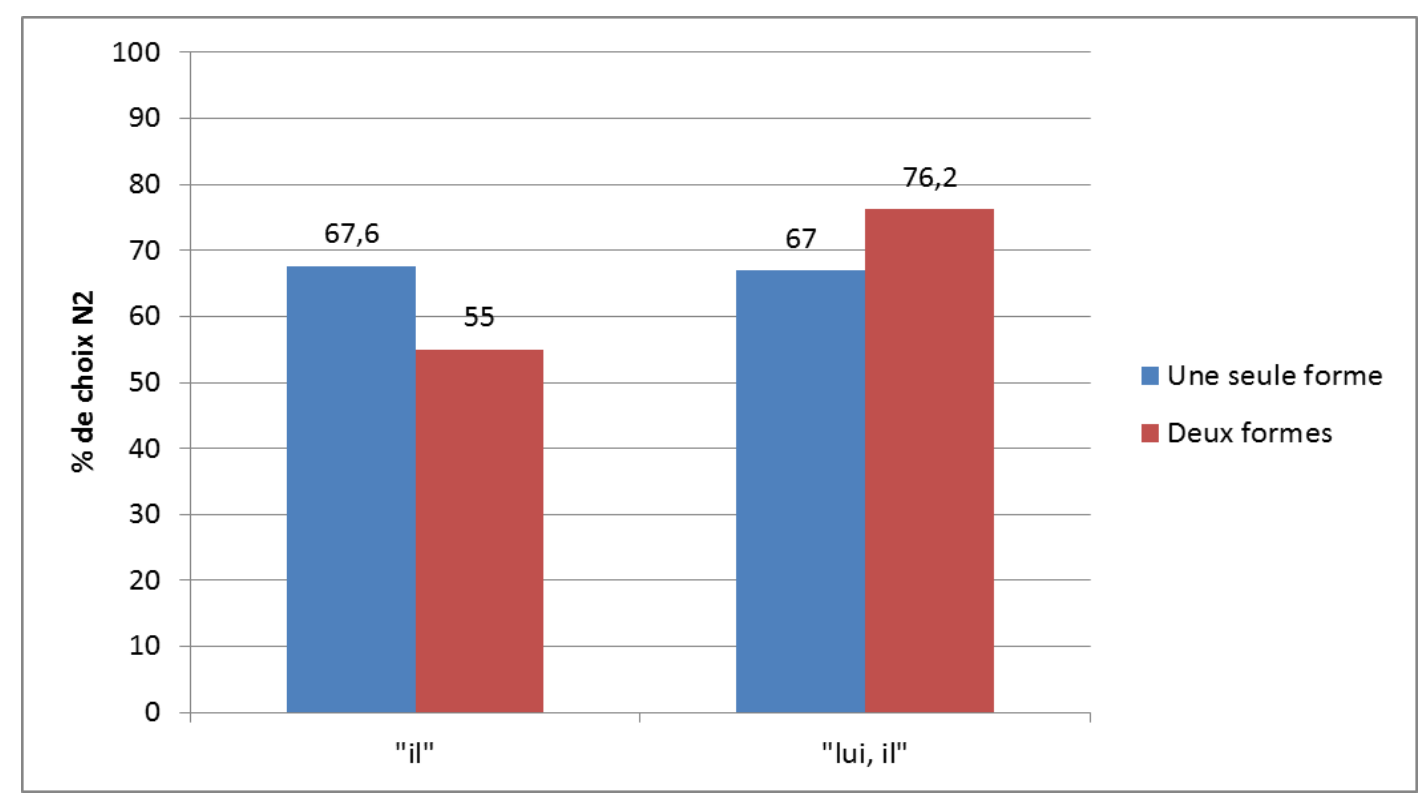

Figure 1. Pourcentages de choix pour N2 suivant le type de forme pronominale («il»vs «lui, il») et le type de questionnaire (une seule forme $v s$ deux formes).

Pour les analyses statistiques, les choix pour N1 vs N2 étaient respectivement codés 0 et 1 . Les réponses qui ne mentionnaient ni N1, ni N2, ont été écartées des analyses (à savoir, 36 des 1624 réponses $(2,2 \%)$ dans le Questionnaire 1, 15 des 1092 réponses (1,4\%) dans le Questionnaire 2, et 8 des 1400 réponses $(0,6 \%)$ dans le Questionnaire 3). Toutes nos analyses ont été réalisées avec des modèles linéaires généralisés à effets mixtes ( $\mathrm{glm}$ et famille binomiale dans R [R Development Core Team, 2013]). Ces modèles permettent d'inclure simultanément les deux effets aléatoires croisés des sujets et des items (Baayen, Davidson \& Bates, 2008 ; Barr, Levy, Scheepers \& Tily, 2013). Nous avons inclus les sujets et les items comme intercepts aléatoires, et toutes les pentes aléatoires que notre plan expérimental permettait (pour une discussion, voir Barr et al., 2013). Dans la suite de la présentation des résultats, nous précisons à chaque fois que le modèle complet n'a pas convergé, ainsi que la simplification de la structure 
des effets aléatoires que nous avons appliquée (voir Barr et al., 2013). ${ }^{\text {iii }}$ Les packages lme4 (Bates, Maechler, Bolker \& Walker, 2015) et languageR (Baayen, 2011) ont été utilisés, les khi-carrés du rapport de vraisemblance et les valeurs-p pour les effets fixes ont été obtenus grâce à la fonction Anova du package $\operatorname{car}$ (Fox \& Weisberg, 2011).

\subsubsection{Analyses inter-questionnaires}

Afin de tester si le fait d'apparaître dans un même questionnaire influençait l'interprétation des deux formes «il »vs «lui, il», nous avons calculé un modèle linéaire généralisé à effets mixtes avec les données des trois questionnaires. La variable dépendante était le choix des participants (N1, N2). Nous avons inclus dans le modèle les variables prédictives «Type de forme pronominale » («il»vs «lui, il ») et «Type de questionnaire» (avec une seule forme vs deux formes) et leur interaction, ainsi que les variables aléatoires sujets et items. Le modèle complet (incluant l'interaction des effets principaux dans les pentes aléatoires des items) n'a pas convergé, probablement à cause de la forte consistance des réponses dans certaines conditions. Plusieurs participants ont en effet systématiquement choisi N2 pour tous les items (surtout dans la condition « lui, il » du Questionnaire 3). Le modèle calculé ne comportait donc que les intercepts aléatoires des sujets et des items. Ce modèle a révélé un effet de l'interaction Type de forme pronominale*Type de questionnaire $\left(\mathrm{X}^{2}(1)=7.2, \mathrm{p}<.01\right)$, un effet du Type de forme pronominale $\left(\mathrm{X}^{2}(1)=73.42, \mathrm{p}<.0001\right)$ et pas d'effet principal du Type de questionnaire $\left(\mathrm{X}^{2}(1)=2.1, \mathrm{p}>\right.$ $.10)$.

Puis, afin de mieux comprendre l'interaction observée, nous avons réalisé des analyses pour chaque type de forme pronominale («il» et «lui, il»). Pour chacune des formes, nous avons utilisé un modèle incluant la variable prédictive «Type de questionnaire» (avec une seule forme $v s$ deux formes), et les variables aléatoires sujets et items. Le facteur «Type de questionnaire » était inclus dans les pentes aléatoires des items. Le modèle pour la forme «il » a révélé un effet significatif du type de questionnaire $\left(\mathrm{X}^{2}(1)=8.73, \mathrm{p}<.01\right)$ : les choix pour $\mathrm{N} 2$ étaient moins fréquents lorsque « il » apparaissait avec la forme «lui, il» (Questionnaire 3) que lorsqu'il apparaissait seul (Questionnaire 1). En revanche, le modèle pour la forme « lui, il » n'a pas révélé d'effet statistiquement significatif du type de questionnaire $\left(\mathrm{X}^{2}(1)=1.47, \mathrm{p}>.10\right)$. On peut cependant noter que la différence numérique observée allait dans le sens attendu : les choix pour N2 étaient plus fréquents lorsque la forme «lui, il » apparaissait avec «il» (Questionnaire 3) que lorsqu'elle apparaissait seule (Questionnaire 2).

\subsubsection{Analyses intra-questionnaires}

Dans les questionnaires 1 et 2, afin de tester si le pourcentage de choix observé pour N2 était significativement différent du hasard, nous avons utilisé un modèle linéaire généralisé à effets mixtes avec les choix (N1, N2) comme variable dépendante, les sujets et les items comme variables aléatoires, et pas de variables prédictives. Si l'intercept aléatoire d'un tel modèle est significatif, cela indique que la préférence pour l'un ou l'autre des choix est significativement différente du hasard. Pour le Questionnaire 1, l'analyse révèle que la préférence pour N2 est significativement différente de $50 \%$ (estimate $=1.15$, $\mathrm{SE}=0.26, \mathrm{z}=4.39, \mathrm{p}<.001$ ). De même, pour le Questionnaire 2, l'analyse révèle que la préférence pour $\mathrm{N} 2$ est significativement différente de $50 \%$ (estimate $=1.54, \mathrm{SE}=0.56, \mathrm{z}=2.76, \mathrm{p}<.01$ ).

Dans le Questionnaire 3, pour tester la différence entre les deux conditions « il » et «lui, il », nous avons utilisé un modèle linéaire généralisé à effets mixtes avec les choix $(\mathrm{N} 1, \mathrm{~N} 2)$ comme variable dépendante, la forme pronominale («il »vs «lui, il ») comme variable prédictive, et les sujets et les items comme variables aléatoires. L'analyse révèle un effet significatif de la forme pronominale (estimate $=1.48, \mathrm{SE}=$ $0.32, \mathrm{z}=4.68, \mathrm{p}<.001)$ : il y a significativement plus de choix pour N2 avec la forme « lui, il » qu'avec le pronom «il ». Nous avons ensuite testé séparément les modèles des sous-ensembles contenant les données de la condition « il » et les données de la condition «lui, il » afin de savoir si, dans chacune de ces conditions, la préférence observée pour N2 était différente du hasard. Pour ce faire, nous avons utilisé des modèles linéaires généralisés à effets mixtes pour chaque sous-ensemble de données, avec les choix $(\mathrm{N} 1, \mathrm{~N} 2)$ comme variable dépendante, les sujets et les items comme variables aléatoires, et pas de 
variables prédictives. Les analyses ont révélé que la préférence pour N2 dans la condition «il » n'était statistiquement pas significative (estimate $=0.24, \mathrm{SE}=0.19, \mathrm{z}=1.32, \mathrm{p}=0.19$ ). Pour la condition « lui, il », il n'a pas été possible d'exécuter le modèle car il ne convergeait pas. Probablement pour les mêmes raisons que vues au $\S 2.2 .1$ (très forte consistance des réponses dans cette condition). Le modèle a convergé lorsque nous avons enlevé les sujets des facteurs aléatoires et a révélé que la préférence pour N2 observée dans la condition « lui, il » était statistiquement différente de 50\% (estimate $=1.16, \mathrm{SE}=0.09$, $\mathrm{z}=12.72, \mathrm{p}<.0001)$.

\section{Discussion}

Nos résultats révèlent d'une part, que les participants ont préférentiellement choisi N2 comme antécédent du pronom (et ce, quel que soit le type de pronom) et d'autre part, que cette préférence était modulée par la présence des deux formes « il » et «lui, il » dans le même questionnaire. Nous observons en effet une interaction entre forme du pronom et contexte : la forme de l'expression anaphorique (plus ou moins réduite) influence son interprétation seulement lorsque les deux formes apparaissent ensemble.

\subsection{Préférence pour N2}

Nous observons une préférence pour le second nom (et objet) mentionné dans la première phrase dans tous les questionnaires et toutes les conditions. Ce résultat peut paraître surprenant si on s'attend à ce que l'anaphore pronominale soit interprétée comme se référant à l'antécédent le plus saillant. En effet, nous avons plusieurs raisons de penser que le premier nom dans la première phrase est l'antécédent le plus saillant puisqu'il est à la fois premier mentionné, sujet, agent et topique. Nous savons cependant que l'interprétation des pronoms anaphoriques est sensible au sémantisme des verbes et, en particulier, à la causalité implicite associée à certains verbes. Dans l'exemple (7a), le verbe «effrayer » est biaisé en faveur de N1 alors que le verbe «craindre » dans l'exemple (7b) est biaisé en faveur de N2. En effet, si on demande de compléter les énoncés (7a) et (7b), la majorité des continuations en (7a) porteront sur «Pierre» alors qu'en (7b) les continuations porteront majoritairement sur «Marie » (Garvey \& Caramazza, 1974 ; Ferstl, Garnham \& Manouilidou, 2011).

$$
\begin{aligned}
& \text { a. Pierre effraie Marie parce qu'.... } \\
& \text { b. Pierre craint Marie parce qu'.... }
\end{aligned}
$$

Ce biais causal a non seulement été observé à la suite de subordonnées causales comme en (7) mais aussi entre deux phrases indépendantes telles que dans nos questionnaires (Kehler, Kertz, Rohde \& Elman, 2008 ; Bott \& Solstad, 2014). Les verbes biaisés en faveur de N2 comme en (7b) font qu'un pronom sujet subséquent sera préférentiellement interprété en faveur du second nom mentionné (et objet) au lieu du premier nom et sujet (voir e.g., Caramazza, Grober, Garvey \& Yates, 1977 ; Garnham \& Oakhill, 1985 ; Vonk, 1985 ; Garnham, Oakhill \& Cruttenden, 1992 ; McKoon, Greene \& Ratcliff, 1993 ; Carreiras, Garnham \& Oakhill, 1996). Il est possible qu'un tel biais causal lié aux verbes utilisés dans notre matériel ait contribué à la préférence pour N2 que nous avons observée. Un inventaire de verbes annotés suivant leur biais causal, comme il en existe déjà pour l'anglais (voir e.g., Ferstl et al., 2011), est en construction pour le français (Mertz, Amsili \& Hemforth, 2015). Malheureusement, tous les verbes utilisés dans nos questionnaires n'ont pas été testés pour cet inventaire. En l'absence de ces données normatives, nous ne pouvons conclure de façon définitive que la préférence pour N2 observée dans les questionnaires 1 et 2 dépend du sémantisme des verbes dans la première phrase.

On peut aussi noter que, contrairement à ce que nous avions pu prédire, la préférence observée dans les questionnaires 1 et 2 , où une seule forme apparaissait, est la même. La préférence pour N2 est particulièrement stable quelle que soit la forme pronominale utilisée, «il» ou «lui, il ». En revanche, cette préférence de base est modifiée par la présence des deux formes dans un même questionnaire. 


\subsection{Influence du contexte expérimental}

C'est à notre avis le résultat le plus intéressant de nos questionnaires : l'interprétation d'une forme pronominale ambiguë est influencée par la présence d'une autre forme pronominale plus ou moins réduite. En effet, on observe que la présence de «lui, il» dans le même questionnaire que le pronom «il», augmente les interprétations du pronom «il» en faveur de N1, i.e. le référent saillant (premier mentionné, sujet et topique) et les interprétations de «lui, il» en faveur de N2, l'antécédent moins saillant. La division fonctionnelle prédite entre la forme pronominale réduite «il » et la forme accentuée « lui, il » apparaît donc seulement lorsque les deux formes sont présentes dans un même questionnaire.

Comme nous avons vu au $\S 1.1$, un effet comparable du contexte expérimental a été observé par de la Fuente et Hemforth (2013) en espagnol. Ils ont en effet observé que la division du travail entre pronoms nuls et pronoms ouverts était accentuée lorsque les deux formes apparaissaient dans un même questionnaire. Ils interprètent cet effet comme étant le résultat de stratégies métalinguistiques développées consciemment par les participants. La tâche off-line de complètement de phrases, utilisée dans notre étude et celle de de la Fuente et Hemforth, laisse en effet la possibilité aux participants de mettre en place des stratégies conscientes. Nous pensons néanmoins que l'influence de la présence des deux formes (plus ou moins réduites) n'est pas nécessairement le résultat de stratégies conscientes. Pour le tester, nous envisageons de réaliser les mêmes manipulations mais avec une technique expérimentale on-line comme l'enregistrement des mouvements oculaires dans le paradigme du monde visuel. Quoi qu'il en soit, ces résultats, observés dans des langues différentes, avec des constructions différentes, relèvent très probablement des mêmes mécanismes (conscients ou pas). L'instruction de traitement associée à une forme anaphorique varie suivant le contexte expérimental. L'instruction de traitement en faveur d'un antécédent saillant sera augmentée en présence d'une forme pronominale moins accentuée; et l'instruction de traitement en faveur d'un antécédent peu saillant d'une forme accentuée augmentera en présence d'une forme réduite.

A notre connaissance, dans toutes les expériences qui testaient la division du travail entre différents marqueurs anaphoriques suivant leur degré d'atténuation, les différentes formes anaphoriques étaient présentées ensemble. En comparant des contextes expérimentaux avec une seule forme vs deux formes, nous venons de voir comment la présentation de formes plus ou moins réduites dans une même expérience influence leur interprétation. Nous ne savons cependant pas quel contexte (avec une seule forme ou deux formes) est le plus écologique. Avec un questionnaire où les deux formes sont présentées, on prend le risque d'attirer exagérément l'attention des participants sur le contraste entre les deux formes. A l'inverse, dans un questionnaire avec une seule forme, on prend le risque que la forme alternative soit sous-représentée par rapport à sa fréquence d'occurrence dans une situation naturelle de communication, minimisant ainsi le contraste entre les deux formes. Si nos résultats ne permettent pas de décider quel contexte est le plus réaliste, ils nous renseignent en revanche sur le rôle des formes alternatives dans le traitement du langage. Ils montrent en effet à quel point les locuteurs peuvent rapidement adapter leur préférence d'interprétation à la présence de formes alternatives dans le contexte (voir aussi, Fine, Jaeger, Farmer \& Qian, 2013). La cooccurrence dans le même contexte de formes alternatives (ici, une forme pronominale réduite et une forme accentuée) modifie leur instruction de traitement et on peut présumer que cet effet s'observe aussi bien dans un contexte expérimental qu'écologique.

\section{Références bibliographiques}

Alonso-Ovalle, L., Fernandes-Solera, S., Frazier, L., \& Clifton, C. (2002). Null vs. overt pronouns and the TopicFocus articulation in Spanish. Rivista di Linguistica, 14(2), 1-19.

Ariel, M. (1990). Accessing noun-phrase antecedents. London: Routledge.

Baayen, R. H. (2011). languageR: Data sets and functions with "analyzing linguistic data: A practical introduction to statistics.” R package version 1.4. Disponible à l'adresse : http://CRAN.R-project.org/package=languageR

Baayen, R. H., Davidson, D. J., \& Bates, D. M. (2008). Mixed-effects modeling with crossed random effects for subjects and items. Journal of Memory and Language, 59, 390-412. 
Barr, D. J., Levy R., Scheepers C., \& Tily H. J. (2013). Random effects structure for confirmatory hypothesis testing: Keep it maximal. Journal of Memory and Language, 68, 255-278. doi:10.1016/j.jml.2012.11.001

Bates, D., Maechler, M., Bolker, B., \& Walker, S. (2015). Fitting Linear Mixed-Effects Models Using lme4. Journal of Statistical Software, 67(1), 1-48. doi:10.18637/jss.v067.i01

Bott, O., \& Solstad, T. (2014). From verbs to discourse - a novel account of implicit causality. In B. Hemforth, B. Schmiedtova, \& C. Fabricius-Hansen (Eds.), Psycholinguistics approaches to meaning and understanding across languages, Studies in theoretical psycholinguistics (pp. 83-109). Munich: Springer.

Brown, R., \& Fish, D. (1983). The psychological causality implicit in language. Cognition, 14, 237-273.

Caramazza, A., Grober, E., Garvey, C., \& Yates, J. (1977). Comprehension of anaphoric pronouns. Journal of verbal learning and verbal behavior, 16(5), 601-609. doi:10.1016/S0022-5371(77)80022-4

Carminati, M. N. (2002). The processing of Italian subject pronouns. PhD dissertation. University of Massachusetts at Amherst.

Carminati, M. N. (2005). Processing reflexes of the Feature Hierarchy (Person > Number > Gender) and implications for linguistic theory. Lingua, 115, 259-285.

Carreiras, M., Garnham, A., \& Oakhill, J. (1996). Understanding anaphora: The role of superficial and conceptual information. In M. Carreiras, J. E. García-Albea, \& N. Sebastián-Gallés (Eds.), Language processing in Spanish (pp. 241-274). Mahwah, NJ: Erlbaum.

Costa, A. (2010). Referential chain's processing. In A. França \& M. Maia (Eds.) Papers in Psycholinguistics Proceedings of the First International Psycholinguistics Congress of ANPOLL (pp. 291-311). Rio de Janeiro: Imprinta.

Costa, A., Faria, I. H., \& Kail, M. (2004). Semantic and Syntactic Cues'Interaction on Pronoun Resolution in European Portuguese. In A. Branco, T. McEnery and T. Mitkov (Eds.), DAARC 2004, Proceedings of 5th Discourse Anaphora Resolution Colloquium (pp. 45-50). Lisboa: Ed. Colibri.

Drummond, A. (2010). Internet Based EXperiments (IBEX) (version 0.3). Disponible à l'adresse : http://spellout.net/ibexfarm/

Ferstl, E. C., Garnham, A., \& Manouilidou, C. (2011). Implicit causality bias in English: A corpus of 300 verbs. Behavior Research Methods, 43(1), 124-135. doi:10.3758/s13428-010-0023-2

Filiaci, F. (2010). Null and overt subject biases in Spanish and Italian: a cross-linguistic comparison. In Borgonovo, C., Español-Echevarría, M. \& Prévost, P. (Eds.), Selected Proceedings of the 12th Hispanic Linguistic Symposium (pp. 171-182). Somerville, MA: Cascadilla Proceedings Project.

Filiaci, F., Sorace, A., \& Carreiras, M. (2013). Anaphoric biases of null and overt subjects in Italian and Spanish: a cross-linguistic comparison. Language, Cognition, and Neuroscience, 29, 825-843.

Fine, A. B., Jaeger, T. F., Farmer, T., \& Qian, T., (2013). Rapid Expectation Adaptation During Syntactic Comprehension. PLoS ONE, 8. doi:10.1371/journal.pone.0077661

Fossard, M., \& Rigalleau, F. (2005) Referential accessibility and anaphor resolution: the case of the french hybrid demonstrative pronoun celui-ci / celle-ci. In A. Branco, T. McEnery \& R. Mitkov (Eds.). Anaphora Processing: Linguistic, Cognitive and Computational Modeling (pp. 283-300). Amsterdam: John Benjamins Publishing Company.

Fox, J., \& Weisberg, S. (2011). An R Companion to Applied Regression (Second ed.). Thousand Oaks CA: Sage.

Frazier, L. (2012). Squib: Co-reference and adult language comprehension. Revista Do Programa De Pos-Graduacao Em Linguistica Da Universidade Federal Do Rio De Janeiro, 1-11.

de la Fuente, I., \& Hemforth, B. (2013). Effects of Clefting and Left-Dislocation on Subject and Object Pronoun Resolution in Spanish. In J. Cabrelli Amaro et al. (Eds.), Selected Proceedings of the 16th Hispanic Linguistics Symposium (pp. 27-45). Somerville, MA: Cascadilla Proceedings Project.

de la Fuente, I., Hemforth, B., Colonna S, \& Schimke, S. (sous presse). The role of syntax, semantics, and pragmatics in pronoun resolution: A cross-linguistic overview. In A. Holler, C. Goeb, \& M. Ellert (Eds.), Experimental Perspectives on Anaphora Resolution. Series "Linguistische Arbeiten”, de Gruyter. 
Garnham, A., \& Oakhill, J. (1985). On-line resolution of anaphoric pronouns: Effects of inference making and verb semantics. British Journal of Psychology, 76(3), 385-393.

Garnham, A., Oakhill, J., \& Cruttenden, H. (1992). The role of implicit causality and gender cue in the interpretation of pronouns. Language and Cognitive Processes, 7, 231-255.

Garvey, C., \& Caramazza, A. (1974). Implicit causality in verbs. Linguistic Inquiry, 5(3), 459-464.

Givón, T. (1983). Topic continuity in discourse: A quantitative cross-language study. Philadelphia \& Amsterdam: John Benjamins.

Gundel, J., Hedberg, N., \& Zacharski, R. (1993). Cognitive status and the form of referring expressions in discourse. Language, 69(2), 274-307.

Jegerski, J., VanPatten, B., \& Keating, G. D. (2011). Cross-linguistic variation and the acquisition of pronominal reference in L2 Spanish. Second Language Research, 33(2), 193-221.

van Kampen, N. J. (2008). Anaphoric pronouns for topic devices. In V. Stojanovik \& T. Marinis (Eds.), Proceedings of the Child Language Seminar 30 (pp. 67-77). Reading UK: University of Reading.

Keating, G.D., VanPatten, B., \& Jegerski, J. (2011). Who was walking on the beach? Anaphora resolution in Spanish heritage speakers and adult second language learners. Studies in Second Language Acquisition, 33(2), 193-221.

Kehler, A., Kertz, L., Rohde, H., \& Elman, J. L. (2008). Coherence and coreference revisited. Journal of Semantics, 25(1), 1-44. doi:10.1093/jos/ffm018

Luján, M. (1985). Binding properties of overt pronouns in null pronominal languages. In W. Eilfort, P. Kroeber \& K. Peterson (Eds.), Proceedings of the 21st Regional Meeting of the Chicago Linguistic Society (pp. 424-438). Chicago: University of Chicago.

Luján, M. (1986). Stress and binding of pronouns. In A. Farley, P. Farley \& K.-E. McCullough (Eds.), Papers from the parasession on pragmatics and grammatical theory (pp. 248-262). Chicago: University of Chicago.

McKoon, G., Greene, S. B., \& Ratcliff, R. (1993). Discourse models, pronoun resolution, and the implicit causality of verbs. Journal of Experimental Psychology: Learning, Memory, and Cognition, 19, 1040-1052.

Mertz, J., Amsili, P., \& Hemforth B. (2015). Implicit causality bias in French: An inventory of 300 verbs. Manuscrit en préparation.

R Development Core Team. (2013). R: A Language and Environment for Statistical Computing [Computer software manual]. Vienna, Austria. Disponible à l'adresse: http://www.R-project.org/ (Version 3.0.0).

Vonk, W. (1985). The immediacy of inferences in the understanding of pronouns. In G. Rickheit \& H. Strohner (Eds.), Inferences in text processing (pp. 205-218). Amsterdam: Elsevier Science.

i Pour une discussion de la différence de traitement des pronoms ouverts en italien et en espagnol, voir Filiaci et al. (2013).

ii Nous avons choisi de ne présenter dans nos items que des antécédents masculins afin d'éviter la forme « elle, elle » trop inhabituelle à l'écrit et, à notre avis, difficile a interpréter sans la prosodie qui l'accompagne.

iii De plus, à chaque fois que pour des raisons de non-convergence, ce n'est pas le modèle complet qui a été retenu, les conclusions des modèles logistiques mixtes ont été supportées par des analyses par sujets et par items séparées que nous ne reportons pas ici pour des raisons de lisibilité. 